\title{
PREPARACIÓN DEPORTIVA EN EL GOALBALL: UNA REVISIÓN SISTEMÁTICA
}

\section{SPORTS PREPARATION IN GOALBALL: A SYSTEMATIC REVIEW}

\author{
Mónica Paulina Fernández Muñoz ${ }^{1,2}$, Kevin Campos Campos ${ }^{2}$, Cristian Luarte Rocha ${ }^{2,3}$ \\ y Luis Felipe Castelli ${ }^{2,4}$ \\ monicafernandez@unach.cl; kevincamposcampos@gmail.com; cristian.luarte@uss.cl; \\ lcastelli@ubiobio.cl \\ ${ }^{1}$ Universidad Adventista de Chile, Chillán, Chile. \\ ${ }^{2}$ Grupo de Investigación en Deporte Adaptado y Paralímpico - GIDEPAUSS, Concepción, \\ Chile. \\ ${ }^{3}$ Universidad San Sebastián, Concepción, Chile. \\ ${ }^{4}$ Universidad del Bío Bío, Chillán, Chile.
}

Envío original: 2021-02-04 Reenviado: 2021-04-12 Aceptado: 2021-04-14

Publicado: 2021-05-04

Doi: https://doi.org/10.15517/pensarmov.v19i1.45702

\begin{abstract}
RESUMEN
El objetivo del estudio fue describir el conocimiento científico producido en los últimos 10 años, respecto a la preparación deportiva (PD) con énfasis en el sistema de entrenamiento en atletas de Goalball de diferentes niveles competitivos. Para eso, fue realizada una revisión sistemática en las principales bases de datos: Web Of Science, Scopus, PubMed, SciELO y LILACS entre enero de 2010 hasta enero de 2021. Se identificó un total de 136 estudios donde posterior a la eliminación de duplicados, aplicación de los criterios de exclusión e inclusión, conforme procedimientos presentados en el flujograma PRISMA, se seleccionaron 15 artículos originales para su análisis cualitativo. Los principales resultados evidenciaron que el metabolismo anaeróbico aláctico es determinante en la modalidad, existiendo una fuerte relación entre la fuerza/potencia de extremidades superiores e inferiores con el lanzamiento del balón. Por otro lado, existen patrones repetitivos en la técnica de lanzamiento según género, así como también efectividad según tipo de lanzamiento, recalcando que la velocidad del lanzamiento es necesaria para la consecución
\end{abstract}


de un gol. De igual forma, existen funciones predominantes según las posiciones dentro del campo de juego que son necesarias considerar. Finalmente, el entrenamiento mental favorece la adquisición de técnicas que ayudan a superar obstáculos imprevistos durante una competición. Se observa la necesidad de mayores evidencias y conocimientos acerca de la PD en dicha modalidad ya que constituyen factores imprescindibles que deben ser contemplados por los entrenadores, en función de optimizar el rendimiento competitivo de sus atletas.

Palabras clave: preparación deportiva, Goalball, entrenamiento de atletas, revisión sistemática

\begin{abstract}
The objective of the study was to describe the scientific knowledge produced in the last 10 years, regarding sports preparation (PD) with an emphasis on the training system in Goalball athletes of different competitive levels. For this, a systematic literature review was carried out in the main databases: Web of Science, Scopus, PubMed, SciELO and LILACS between January 2010 and January 2021. A total of 136 studies were identified where after the elimination of duplicates, application of the exclusion and inclusion criteria, according to procedures presented in the PRISMA flow chart, 15 original articles were selected for qualitative analysis. The main results showed that alactic anaerobic metabolism is decisive in the modality, with a strong relationship between the strength / power of the upper and lower extremities with the launch of the ball. On the other hand, there are repetitive patterns in the throwing technique according to gender, as well as effectiveness according to the type of throw, emphasizing that the speed of the throw is necessary to achieve a goal. Similarly, there are predominant functions according to the positions within the playing field that need to be considered. Finally, mental training favors the acquisition of techniques that help overcome unforeseen obstacles during a competition. The need for more evidence and knowledge about PD in this modality is observed since they constitute essential factors that must be considered by coaches, in order to optimize the competitive performance of their athletes.
\end{abstract}

Keywords: sports preparation, Goalball, athlete training, systematic review 


\section{INTRODUCCIÓN}

El Goalball fue creado en 1946 por el austriaco Hanz Lorenzen y el alemán Sett Reindle, en un esfuerzo por ayudar en la rehabilitación a veteranos de guerra que, debido a conflictos en zonas de combate, padecieron de discapacidad visual (IBSA, 2020). La popularidad y él interés por la práctica del deporte por las personas con discapacidad visual, permitió su desarrollo a lo largo de todo el mundo, pasando a integrar los Juegos Paralímpicos de Verano, siendo la categoría masculina incluida desde los Juegos de 1976 en Toronto y la categoría femenina desde los Juegos de Nueva York 1984 (Maturana et al., 2005; Monreal, 2010 citado por Morato y Gavião, 2012; Parsons y Winckler, 2012). La International Blind Sports Federation - IBSA, fue creada en 1981 y, desde entonces, es la organización internacional que gerencia la modalidad, responsable por realizar los campeonatos mundiales y continentales (IBSA, 2020).

La elegibilidad para competir en torneos oficiales es otorgada para atletas con clasificación oftalmológica agrupados en la clase B1 (considerado como ciegos), B2 o B3 (considerados baja visión) que compiten entre sí, con el uso obligatorio de una venda, con el objetivo de reducir ventajas de los atletas que presentan menor comprometimiento visual, tornando la competencia más justa (IBSA, 2020).

El partido se realiza en una cancha de juego con $18 \mathrm{~m}$ de largo y $9 \mathrm{~m}$ de ancho, generalmente en gimnasios cubiertos y con el menor ruido posible. Es considerada una modalidad colectiva de oposición sin invasión, ya que el juego se realiza con tres atletas para cada equipo con el objetivo de realizar el "gol" por medio de lanzamientos del balón (realizados desde su sector defensivo) mientras que el equipo adversario (en su sector defensivo) intenta evitar que el balón ultrapase la línea del gol. El balón contiene cascabeles en su interior que produce sonido cuando está en movimiento y contacto con el suelo, aspecto que facilita a los atletas identificar la posición del balón y su orientación durante el juego (Da Rocha, 2007, Morato y Gavião, 2012).

En su inicio, la IBSA presentaba 30 países afiliados y actualmente presenta 94 países renqueados que disputan los principales torneos de la modalidad en la categoría masculina y 58 países en la categoría femenina (IBSA, 2020). El crecimiento de la modalidad representada por el número de países y atletas durante los principales torneos internacionales (IBSA, 2020), así como de estudios con el objetivo de producir conocimientos acerca de la modalidad (Morato, Menezes, Fonseca y da Cunha Furtado, 2018), pueden considerarse como puntos claves para el aumento de la competitividad a lo largo de los años, lo que impacta directamente en cambios en la preparación deportiva de 
los atletas con el objetivo de lograr un desempeño óptimo durante la competencia (Campos et al., 2019; Petrigna et al., 2020).

Gomes (2009) manifiesta que la Preparación Deportiva (PD) se compone de tres sistemas que, de una forma integrada, objetivan alcanzar un estado óptimo de desempeño posibilitando mejores oportunidades para el éxito durante las competencias. Estos sistemas son (1) Sistema de Competición, referido a todas las manifestaciones de la competición, desde su forma de disputa, hasta el diagnóstico de las acciones motoras realizadas por el atleta en actividades competitivas, (2) El Sistema de Factores Complementarios que trata de todos los medios que auxilian la preparación del atleta, principalmente relacionados con la recuperación post carga de entrenamiento y con los medios utilizados para la movilización del atleta para la competencia, y que incluye, entre otras áreas, la psicología, medicina, fisioterapia, nutrición y masoterapia; finalmente, (3) el Sistema de entrenamiento, que se relaciona con el desarrollo y perfeccionamiento de las capacidades y aspectos fundamentales para el óptimo desempeño deportivo.

Respecto a lo último, diversos autores (Matveev, 1996; Platonov, 2001; Platonov, 2008; Gomes, 2009; Moreira, 2010; Campos et al., 2019; Santos, 2019; Souza, Barra et al., 2020) reafirman que el sistema de entrenamiento presenta particularidades y especificidades de un deporte a otro, por lo que, en general, se consideran 4 componentes dentro de este sistema, siendo la 1) Preparación Física, que contempla los entrenamientos de resistencia, entrenamientos de la capacidad de fuerza, velocidad, flexibilidad, coordinación y control del estado de preparación física, la 2) Preparación Técnica, define características de la técnica, la preparación de un atleta para asimilar una acción motora, las etapas de la enseñanza de la técnica de una acción motora, la integración de la preparación técnica - física y el control del estado de la preparación técnica, la 3) Preparación táctica del atleta, referido a la comprensión de las tácticas y estrategias, las características generales de las tácticas en diversas modalidades deportivas, los procesos de perfeccionamiento y control de la preparación táctica y la 4) Preparación psicológica, que propone obtener información sobre las competiciones, los oponentes, el nivel de entrenamiento del deportista y su estado en la determinada etapa de la preparación, la determinación de objetivos en las competencias y la estimulación de reglas personales y los motivos generales. Se entrega suficiente información al deportista para superar con éxito la competencia, obteniendo un máximo rendimiento posible.

Sumado a eso, Santos (2019) y Souza, Barra et al. (2020) describen que, en el contexto paralímpico, los aspectos que incorpora la PD son transversales, siendo 
importante reflexionar sobre la especificidad de la modalidad, considerando la deficiencia y la clasificación deportiva que presente el atleta, puesto que, impactará directamente en los medios y métodos de preparación de esos atletas.

Hasta este momento en el Goalball, no fueron identificados estudios que consideren el sistema de entrenamiento en su integralidad, lo que produce conocimientos aislados de variables determinantes para la modalidad, así como no se han identificado registros de una revisión sistemática que englobe los factores asociados al sistema de entrenamiento de atletas de Goalball en nivel competitivo, en las bases consultadas.

En ese sentido, este estudio busca describir las evidencias científicas desarrolladas en la última década (2010-2020) respecto al sistema de entrenamiento (aspectos físicos, técnicos, tácticos y psicológicos) en atletas de Goalball de diferentes niveles competitivos, y así, generar información relevante para los profesionales que participan directamente con la modalidad, tanto en la preparación de nuevos atletas como en el desarrollo de jugadores ya experimentados.

\section{METODOLOGÍA}

\section{Fuentes de datos y búsqueda}

Se realizó una búsqueda desde enero de 2010 hasta enero del 2021, utilizando las bases de datos electrónicas de: Web of Science, Scopus, PubMed, SciELO y LILACS, considerando cuatro operaciones de búsqueda, siendo él termino clave "Goalball" combinado con los términos (Physical performance, Technique or Technical, Tactic or Tactical, Psychology) por medio de los operadores booleanos "AND” y "OR" en dos casos, por ejemplo: "Goalball AND (technique OR technical)". En el caso de idioma español, se usó el descriptor clave "Goalball" combinado con los descriptores (Rendimiento físico, Técnica, Táctica, Psicología), utilizando los mismos operadores booleanos.

\section{Criterios de inclusión}

Los artículos de investigación que cumplieron con los criterios de inclusión y exclusión fueron seleccionados para revisión y aplicación. Se utilizó la estrategia PICOS, la cual representa el acrónimo en inglés de Population, Intervention, Comparison, Outcomes, Study-desing. Para efectos de nuestra investigación, "Population" hace referencia a atletas con discapacidad visual, "Intervention" a la modalidad de Goalball, "Comparison" (no aplica), "Outcomes" enfocado a descripción de aspectos físicos, técnicos, tácticos y 
psicológicos en Goalball y "Study-desing" incluidos aquellos estudios descriptivos y experimentales. Estos elementos que componen dicha estrategia (PICOS), son fundamentales para la formulación de la pregunta de investigación y búsqueda bibliográfica (Roever, 2017; Da Costa Santos, de Mattos Pimenta y Nobre, 2007).

Los estudios fueron incluidos si, (i) estaban en idioma inglés, español o portugués, (ii) fueron publicados entre el 2010 - 2020, (iii) solo eran artículos científicos originales, (iv) la muestra consistía en atletas de género femenino y masculino, de nivel regional, nacional e internacional que practicaban Goalball, ( $v$ ) eran estudios de carácter descriptivos y experimentales, y que (vi) en sus resultados, trataban sobre el rendimiento físico, técnica o táctica y factores psicológicos asociados al rendimiento de la modalidad.

\section{Criterios de exclusión}

Como criterio de exclusión, se eliminaron todos los estudios de revisiones, metaanálisis, resúmenes, declaraciones, artículos de opinión, citas de conferencias científicas, comentarios, editoriales, reseñas de libros, libros, cartas y artículos de revistas no revisados por pares.

\section{Selección de estudios}

El proceso de selección fue realizado por dos revisores, quienes de forma independiente evaluaron los estudios identificados. No fue necesario consultar a un tercer revisor debido a que no hubo discrepancias. Los artículos encontrados en primera instancia, fueron seleccionados en función del título y resumen; cuando el título y resumen fueron relevantes con el tema de investigación, se leyó de manera detallada su texto completo para decidir su inclusión definitiva en esta revisión sistemática. Los artículos seleccionados se ingresaron a una planilla Microsoft Excel ${ }^{\circledR} 2019$ (v.19.0) (Microsoft Corp; Redmond, Washington, EE.UU) con la finalidad de extraer la siguiente información: autor y año de publicación, tamaño de la muestra, objetivo, metodología y resultados. Todos los datos se resumieron mediante tablas descriptivas (tablas $\underline{1}, \underline{2}, \underline{3}$ y $\underline{4}$ ) y se analizaron mediante una síntesis cualitativa. 


\section{RESULTADOS}

\section{Búsqueda de Literatura}

En el diagrama de flujo se muestra la cantidad de artículos incluidos y excluidos en cada fase. Inicialmente se identificó un total de 136 estudios. Posterior a la eliminación de duplicados, se analizaron 76 estudios en búsqueda de contenido relevante mediante título y resumen, excluyendo 56 estudios, quedando 20 artículos para su lectura completa. Finalmente, se excluyeron 5 artículos, los cuales correspondían a estudios fuera del rango de años de publicación $(n=1)$, ensayos $(n=2)$ y revisiones sistemáticas $(n=2)$. Por tanto, se seleccionaron 15 artículos originales para su análisis cualitativo (ver Figura 1).
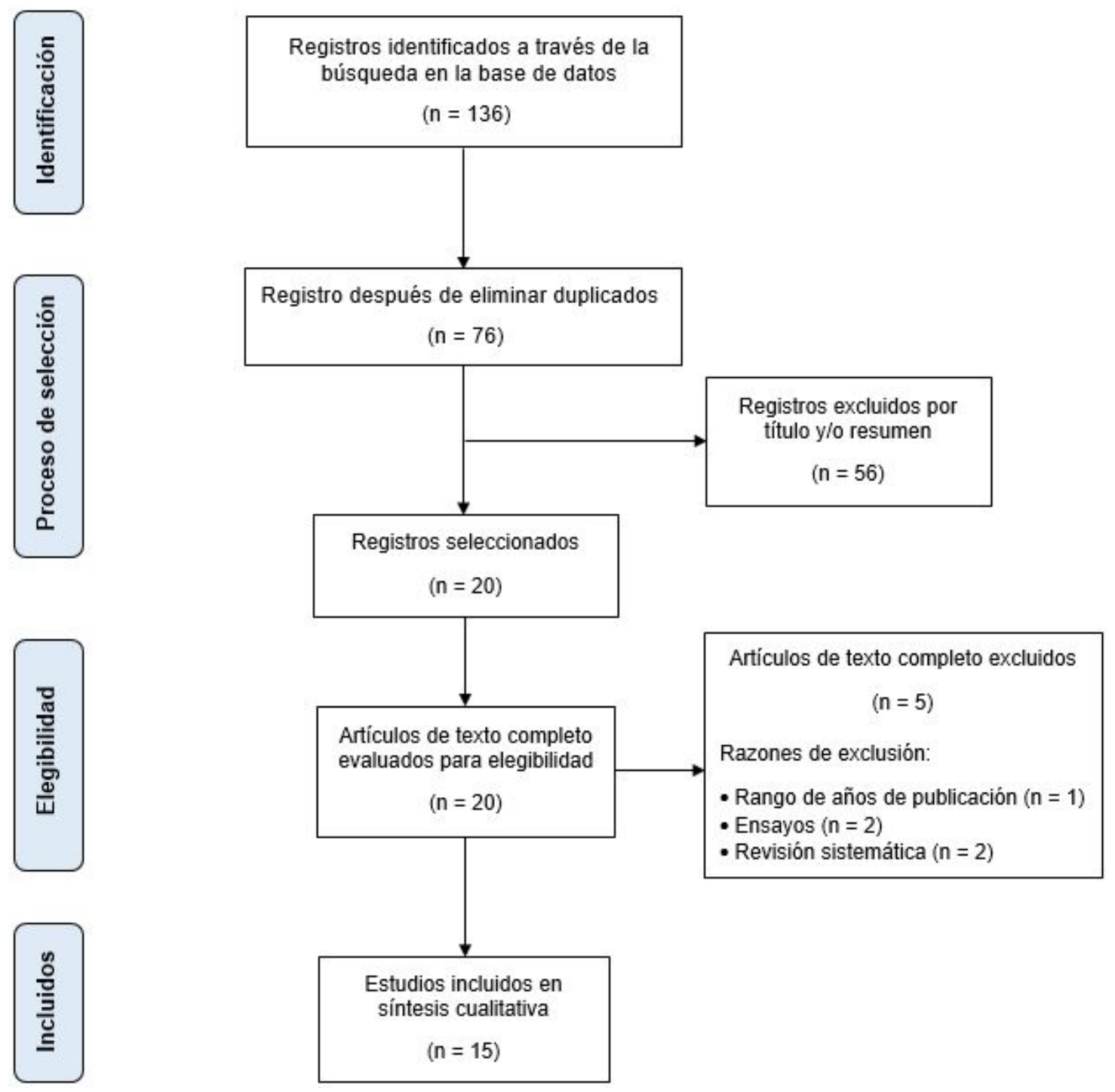

Figura 1. Flujograma PRISMA de artículos incluidos en la revisión. Fuente: Elaboración propia con base en Moher et al. (2009) 


\section{Resultados de Artículos seleccionados}

En las Tablas $\underline{1}, \underline{2}, \underline{3}$ y $\underline{4}$ son presentados los estudios seleccionados y clasificados según los componentes del sistema de entrenamiento, que es parte fundamental de toda PD, evidenciando nueve artículos referidos a los aspectos Físicos $(n=9)$, tres artículos a los aspectos Técnicos $(n=3)$, tres artículos a los aspectos Tácticos $(n=3)$ y un artículo a los aspectos Psicológicos $(n=1)$ en Goalball. 
Tabla 1.

Artículos que presentaron Aspectos Físicos en Goalball

\begin{tabular}{|c|c|c|c|c|}
\hline Autor & Muestra & Objetivo & Metodología & Resultado \\
\hline $\begin{array}{l}\text { Silva, Pereira, } \\
\text { Deprá y Gorla } \\
(\underline{\underline{2010})}\end{array}$ & $\begin{array}{l}9 \text { atletas de Goalball, } \\
\text { sexo: femenino y } \\
\text { masculino. }\end{array}$ & $\begin{array}{l}\text { Evaluar la influencia del } \\
\text { tiempo de reacción (TRe) } \\
\text { en la eficiencia de las } \\
\text { acciones de defensa en } \\
\text { los III Juegos Mundiales } \\
\text { IBSA, } 2007\end{array}$ & $\begin{array}{l}\text { Análisis de Aspectos físicos } \\
\text { del Goalball (tiempo de } \\
\text { reacción) a través de } \\
\text { observación de Video }\end{array}$ & $\begin{array}{l}\text { - Repetición de movimientos y } \\
\text { disposición para la acción influyó en } \\
\text { una mejor respuesta en relación a la } \\
\text { tarea, disminuyendo en el TRe. } \\
\text { - Sin diferencia en acciones (defensa } \\
\text { o gol) en el TRe. }\end{array}$ \\
\hline $\begin{array}{l}\text { Gulick y } \\
\text { Malone (2011) }\end{array}$ & $\begin{array}{l}9 \quad \text { atletas } \\
\text { norteamericanos del } \\
\text { sexo Femenino. }\end{array}$ & $\begin{array}{l}\text { Establecer el contenido, } \\
\text { validez concurrente y } \\
\text { predictiva de una prueba } \\
\text { de sonido modificada } \\
\text { para evaluar el aspecto } \\
\text { físico de aptitud } \\
\text { cardiovascular. }\end{array}$ & $\begin{array}{l}\text { Correlación entre prueba } \\
\text { estandarizada en bicicleta } \\
\text { ergométrica y una prueba de } \\
\text { sonido modificada. }\end{array}$ & $\begin{array}{l}\text { - Fuerte correlación ( } r=0,77 \text { ) entre } \\
\text { bicicleta ergómetro y prueba de } \\
\text { sonido }\end{array}$ \\
\hline $\begin{array}{l}\text { Valdés, Godoy } \\
\text { y } \quad \text { Herrera } \\
(\underline{2014)}\end{array}$ & $\begin{array}{l}14 \text { atletas } \text { de } \\
\text { Goalball, del sexo } \\
\text { masculino }\end{array}$ & $\begin{array}{l}\text { Medir el somatotipo, } \\
\text { composición corporal, } \\
\text { estado nutricional y } \\
\text { condición física. }\end{array}$ & $\begin{array}{l}\text { Protocolo ISAK, pruebas } \\
\text { físicas (abdominales cortos, } \\
\text { salto largo a pies juntos, } \\
\text { flexión y extensión de codos, } \\
\text { flexión de tronco y prueba } \\
\text { Cafra. }\end{array}$ & $\begin{array}{l}\text { - Atletas presentaron } 28,8 \pm 8,2 \% \text { de } \\
\text { GC con un somatotipo mesomorfo } \\
(5,1 \pm 1,11) \text {. } \\
\text { f Valores favorables para } \\
\text { flexibilidad, abdominales y test de } \\
\text { cafra y no satisfactorios para salto } \\
\text { largo y flexo extensión de codos. }\end{array}$ \\
\hline $\begin{array}{l}\text { Molik et al. } \\
(\underline{\underline{2015})}\end{array}$ & $\begin{array}{l}27 \text { atletas } \\
\text { participantes de los } \\
\text { juegos paralímpicos } \\
\text { de Londres 2012. } \\
\text { Sexo: masculinos }\end{array}$ & $\begin{array}{l}\text { (1) Describir el } \\
\text { rendimiento del juego en } \\
\text { función del grado de } \\
\text { discapacidad visual, y (2) } \\
\text { determinar si el } \\
\text { rendimiento del juego } \\
\text { está relacionado con las } \\
\text { características } \\
\text { antropométricas. }\end{array}$ & $\begin{array}{l}\text { Medición de masa corporal, } \\
\text { altura del cuerpo, } \\
\text { envergadura y longitud del } \\
\text { cuerpo en posición defensiva. } \\
\text { Analizaron } 38 \text { partidos de } \\
\text { Goalball. }\end{array}$ & $\begin{array}{l}\text { - B2 y B3 lograron niveles más altos } \\
\text { de efectividad en ataque } \\
(4,28 \pm 2,31 \%) \text {. } \\
\cdot \text { B2 y B3 cometieron mayor número } \\
\text { de infracciones personales } \\
(0,86 \pm 0,65) \text {. } \\
\text { B1 defendieron con más frecuencia } \\
\text { que sus compañeros B2 y B3 } \\
(28.71 \pm 5.89 \text { vs } 23.81 \pm 5.19) \text {. } \\
\text { - Las variables antropométricas no } \\
\text { influyen en el rendimiento } \\
\text { competitivo. }\end{array}$ \\
\hline
\end{tabular}


Continuación de la tabla 1

\begin{tabular}{|c|c|c|c|c|}
\hline Autor & Muestra & Objetivo & Metodología & Resultado \\
\hline $\begin{array}{l}\text { Bednarczuk } \\
\text { al. (2017) }\end{array}$ & $\begin{array}{l}42 \text { atletas } \\
\text { masculinos y } \\
23 \text { atletas } \\
\text { femeninas. }\end{array}$ & $\begin{array}{l}\text { Evaluar niveles de } \\
\text { equilibrio estático } \\
\text { teniendo en cuenta la } \\
\text { excelencia deportiva, } \\
\text { experiencia y cargas } \\
\text { de entrenamiento. }\end{array}$ & $\begin{array}{l}\text { Equilibrio estático se evaluó } \\
\text { mediante } \\
\text { estabilográfica en siguientes } \\
\text { pruebas: pie individual y sobre } \\
\text { ambos pies, con los ojos abiertos } \\
\text { y cerrados. } \\
\text { Se midieron las variables de talla } \\
\text { (cm) y masa corporal }(\mathrm{kg}) \text {. }\end{array}$ & $\begin{array}{l}\text { - Diferencias significativas entre los } \\
\text { competidores que participaron en } \\
\text { rondas preliminar y final. } \\
\text { - Mayor equilibrio estático en atletas } \\
\text { con experiencia hasta } 10 \text { años. } \\
\text { - Los jugadores que participaron en } \\
\text { fase final demostraron mayor } \\
\text { estabilidad postural. }\end{array}$ \\
\hline $\begin{array}{l}\text { Romanov et al. } \\
(\underline{\underline{2017})}\end{array}$ & $\begin{array}{l}22 \text { atletas del } \\
\text { sexo masculino }\end{array}$ & $\begin{array}{l}\text { Determinar las } \\
\text { características } \\
\text { morfológicas y si } \\
\text { existe una correlación } \\
\text { entre indicadores } \\
\text { morfológicos con los } \\
\text { resultados de } \\
\text { competencia. }\end{array}$ & $\begin{array}{l}\text { Evaluación de altura corporal } \\
(\mathrm{BH}) \text {, peso corporal (BW), } \\
\text { circunferencia de cintura (WC), } \\
\text { circunferencia de caderas (HC), } \\
\text { proporción circunferencia cadera- } \\
\text { cintura (WHR), porcentaje de } \\
\text { grasa (\% FAT) y el porcentaje de } \\
\text { tejido muscular (\% MCT). } \\
\text { Resultados oficiales de } \\
\text { competencia post final. }\end{array}$ & $\begin{array}{l}\text { - La Altura es importante para definir } \\
\text { el perfil morfológico. } \\
\text { - Correlación entre BH y clasificación } \\
\text { de equipos }(r=0,66) \text {. } \\
\text { - Correlación entre WHR y la } \\
\text { clasificación de los equipos ( } r=0,54) \text {. } \\
\text { - Distribución de grasa corporal es } \\
\text { importante en la definición del perfil } \\
\text { morfológico de los jugadores de } \\
\text { Goalball. }\end{array}$ \\
\hline $\begin{array}{l}\text { Goulart } \\
(\underline{2018)}\end{array}$ & $\begin{array}{l}7 \text { atletas } \\
\text { masculinos y } \\
4 \quad \text { atletas } \\
\text { femeninas. }\end{array}$ & 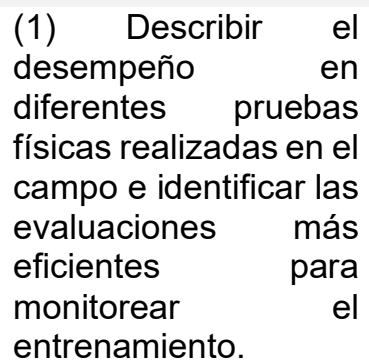 & $\begin{array}{l}\text { Se evaluó el \%GC, fuerza } \\
\text { isométrica máxima de agarre, } \\
\text { salto CMJ, velocidad de } \\
\text { lanzamiento y prueba intermitente } \\
\text { Yo-Yo nivel } 1 .\end{array}$ & $\begin{array}{l}\text { - \%GC con salto CMJ }(r=-0,79) \text { y } \\
\text { YoYo IR1 ( } r=0,-0,90) \text {. } \\
\text { - Salto CMJ y velocidad de } \\
\text { lanzamiento }\left(\mathrm{R}^{2}=\quad 0,57\right) \text {. } \\
\text { - Prensión manual y velocidad del } \\
\text { lanzamiento } \\
\text { - Se sugiere el uso de la prueba CMJ } \\
\text { para el seguimiento de los atletas de } \\
\text { Goalball. }\end{array}$ \\
\hline
\end{tabular}


Continuación de la tabla 1

\begin{tabular}{|c|c|c|c|c|}
\hline Autor & Muestra & Objetivo & Metodología & Resultado \\
\hline $\begin{array}{l}\text { Alves et al. } \\
(\underline{2018)}\end{array}$ & $\begin{array}{l}7 \text { jugadores del } \\
\text { sexo masculino. }\end{array}$ & $\begin{array}{l}\text { (1) Comparar las relaciones } \\
\text { entre parámetros } \\
\text { fisiológicos con juegos } \\
\text { simulados y la relación entre } \\
\text { los parámetros fisiológicos y } \\
\text { el rendimiento técnico del } \\
\text { juego. }\end{array}$ & $\begin{array}{l}\text { (1) prueba incremental para } \\
\text { VO2máx, y esfuerzos } \\
\text { submáximos y supramáximos } \\
\text { para estimar la contribución } \\
\text { anaeróbica máxima. Se } \\
\text { realizaron juegos simulados. }\end{array}$ & $\begin{array}{l}\text { - El VO2máx presentó correlaciones } \\
\text { fuertes entre VO2máx y frecuencia } \\
\text { de lanzamiento ( } r=-0,87) \text { y } \\
\text { recuperación en los juegos ( } r=- \\
0,90) \text {. } \\
\text { - Correlación entre la contribución } \\
\text { anaeróbica aláctica y la densidad de } \\
\text { acciones }(r=0,95) \text {. }\end{array}$ \\
\hline $\begin{array}{l}\text { Campos et al. } \\
(\underline{2020})\end{array}$ & $\begin{array}{l}9 \text { jugadores del } \\
\text { sexo masculino }\end{array}$ & $\begin{array}{l}\text { Identificar la asociación y } \\
\text { relación entre las variables } \\
\text { de fuerza máxima, potencia } \\
\text { muscular, medidas } \\
\text { antropométricas r y } \\
\text { composición corporal con el } \\
\text { lanzamiento del balón. }\end{array}$ & $\begin{array}{l}\text { Medidas antropométricas de } \\
\text { Longitud de Miembros } \\
\text { Superiores (CMS) y } \\
\text { Envergadura (ENV), } \\
\text { Porcentaje Grasa Corporal } \\
\text { (\%GC), prueba 1RM en press } \\
\text { banca, Test de lanzamiento } \\
\text { de balón medicinal, y test de } \\
\text { lanzamiento (TLAN). }\end{array}$ & $\begin{array}{l}\text { - TLAN y 1RMe }(r=-0,80) \text {. } \\
\text { - AMB y 1RMe }(r=0,89) \text {. } \\
\text { - Variables CMS, \%GC y ENV no } \\
\text { fueron determinantes en el } \\
\text { lanzamiento. }\end{array}$ \\
\hline
\end{tabular}

Fuente: Elaboración propia. 
Tabla 2.

Artículos que presentaron Aspectos Técnicos en Goalball

\begin{tabular}{|c|c|c|c|c|}
\hline Autor & Muestra & Objetivo & Metodología & Resultado \\
\hline $\begin{array}{lr}\text { Kornev } & y \\
\text { Pavdrov } & (2017)\end{array}$ & $\begin{array}{l}\text { Final masculina y } \\
\text { femenina del } \\
\text { campeonato } \\
\text { mundial de } \\
\text { Goalball 2014. }\end{array}$ & $\begin{array}{l}\text { Realizar un análisis } \\
\text { completo de las técnicas y } \\
\text { tácticas modernas del } \\
\text { Goalball. }\end{array}$ & $\begin{array}{l}\text { Filmación de la final } \\
\text { del campeonato } \\
\text { mundial } 2014 \text {. }\end{array}$ & $\begin{array}{l}\text { - Jugadores masculinos optaron por los tiros } \\
\left.\text { de rotación de } 360^{\circ} \text { con una mano ( } 82,9 \%\right) \text {. } \\
\text { - Jugadoras femeninas optaron por los tiros } \\
\text { lisos en carrera con una mano }(97,8 \%) \text {. } \\
\text { - Los jugadores centrales (ambos géneros) } \\
\text { dominan en las tácticas defensivas. } \\
\text { - Los jugadores laterales (ambos géneros) } \\
\text { dominan las acciones ofensivas ( } 80,5 \% \text { en } \\
\text { hombres y } 81,3 \% \text { en mujeres). } \\
\text { - Ambos géneros optaron por tiros en } \\
\text { diagonales cortas (47,4\% en hombres y } \\
46,1 \% \text { en mujeres). }\end{array}$ \\
\hline $\begin{array}{l}\text { Link y Weber } \\
(\underline{\underline{2018})}\end{array}$ & $\begin{array}{l}\mathrm{N}=113 \text { partidos } \\
\text { (63 hombres, } 50 \\
\text { mujeres) } \\
\text { Paralímpicos. }\end{array}$ & $\begin{array}{l}\text { Investigar qué tipos de } \\
\text { lanzamientos tienen más } \\
\text { probabilidades de conducir } \\
\text { a un gol. }\end{array}$ & $\begin{array}{lr}\text { Filmación de } & 113 \\
\text { partidos } & (63 \\
\text { hombres, } & 50 \\
\text { mujeres) de las } \\
\text { competiciones } \\
\text { Paralímpicas } 2012, \\
2016 \text { y los } \\
\text { Campeonatos de } \\
\text { Europa 2013 y } 2015 .\end{array}$ & $\begin{array}{l}\text { - Lanzamientos masculinos son más } \\
\text { efectivos hacia el sector lateral en paralelo } \\
(6,6 \%) \text {. } \\
\text { - Lanzamientos femeninos son más efectivos } \\
\text { entre el central y lateral en paralelo }(4,7 \%) \text {. } \\
\text { - Lanzamientos paralelos son más eficaces } \\
\text { en hombres }(4,3 \%) \text { y mujeres }(3,5 \%) \text {. } \\
\text { - Mayor tasa de infracción en tiros planos que } \\
\text { en tiros de rebote, en hombres }(3,2 \%) \text { y } \\
\text { mujeres } \\
\text { - Hombres juegan más tiros con giros } \\
(46,3 \%) \text {. } \\
\text { Mujeres juegan más tiros tradicionales } \\
(203,2 \%) \text {, pero presentan más efectividad en } \\
\text { tiros con giro y rebote }(8,5 \%) \text {. }\end{array}$ \\
\hline $\begin{array}{l}\text { Morato et al. } \\
(\underline{2018})\end{array}$ & $\begin{array}{l}\mathrm{N}=29 \text { atletas de } \\
\text { élite femeninas } \\
\text { (1304 } \\
\text { lanzamientos) } \\
\mathrm{N}=39 \text { atletas de } \\
\text { élite masculinos } \\
\text { (1341 } \\
\text { lanzamientos) }\end{array}$ & $\begin{array}{l}\text { (1) Investigar la influencia } \\
\text { del tiempo, trayectoria y el } \\
\text { tipo de lanzamiento en la } \\
\text { probabilidad de marcar un } \\
\text { gol en partidos femeninos y } \\
\text { masculinos. (2) Categorizar } \\
\text { el tiempo de lanzamiento } \\
\text { del balón, teniendo en }\end{array}$ & $\begin{array}{l}\text { Análisis de video en } \\
20 \text { partidos de } \\
\text { Goalball (diez por } \\
\text { género), } \\
\text { seleccionados al } \\
\text { azar de los Juegos } \\
\text { Paralímpicos de } \\
2008 \text {. }\end{array}$ & $\begin{array}{l}\text { - Mujeres presentan mayor efectividad en } \\
\text { diagonales largas }(4,8 \%) \text { y hombres en } \\
\text { diagonales cortas } \\
\text { - Hombres y mujeres fueron más efectivos } \\
\text { con tiros curvos }(8,3 \% \text { y } 5,2 \% \\
\text { respectivamente). } \\
\text { - En ambos sexos, la reducción del tiempo de } \\
\text { lanzamiento se asoció con una mayor } \\
\text { probabilidad de marcar un gol. }\end{array}$ \\
\hline
\end{tabular}


cuenta la trayectoria y la técnica.
- El tiro con rebote en diagonal corta, fue más veloz en mujeres $(<0,83 \mathrm{~s})$ y el tiro liso en paralelo y diagonal corta en hombres $(<0,53 \mathrm{~s})$.

\section{Fuente: Elaboración propia.}


Tabla 3.

Artículos que presentaron Aspectos Tácticos en Goalball

\begin{tabular}{|c|c|c|c|c|}
\hline Autor & Muestra & Objetivo & Metodología & Resultado \\
\hline $\begin{array}{l}\text { Morato, } \\
\text { Simöes y } \\
\text { Gavião (2012) }\end{array}$ & $\begin{array}{l}\mathrm{N}=20 \text { partidos de los } \\
\text { juegos paralímpicos. } \\
\mathrm{N}=10 \text { partidos } \\
\text { masculinos. } \\
\mathrm{N}=10 \text { partidos } \\
\text { femeninos. }\end{array}$ & $\begin{array}{lll}\text { Interpretar patrones } & \text { y } \\
\text { procesos } & & \\
\text { autoorganizados de la } \\
\text { modalidad. }\end{array}$ & $\begin{array}{l}\text { Filmación de } 20 \\
\text { juegos de los Juegos } \\
\text { Paralímpicos } 2008 .\end{array}$ & $\begin{array}{l}\text { - El ciclo de defensa se equilibra mediante } \\
\text { retroalimentación negativa. Cuando se } \\
\text { produce un gol, infracción, penalti, etc. } \\
\text { Presenta oscilación que, mediante } \\
\text { retroalimentación positiva, puede llevar a un } \\
\text { punto de bifurcación en el que se pueden } \\
\text { asumir nuevos estándares organizativos. } \\
\text { - El sistema de juego de Goalball está } \\
\text { representado por el conjunto de secuencias } \\
\text { (ataque de un equipo / defensa por otro / } \\
\text { resultado). }\end{array}$ \\
\hline
\end{tabular}

\begin{tabular}{|c|c|c|c|c|}
\hline $\begin{array}{lr}\text { Kornev } \quad y \\
\text { Pavdrov (2017) }\end{array}$ & $\begin{array}{l}\text { Final masculina y } \\
\text { femenina del } \\
\text { campeonato mundial } \\
\text { de Goalball } 2014 .\end{array}$ & $\begin{array}{l}\text { Realizar un análisis } \\
\text { completo de las técnicas } \\
\text { y tácticas modernas de } \\
\text { Goalball. }\end{array}$ & $\begin{array}{l}\text { Filmación de la final } \\
\text { del campeonato } \\
\text { mundial } 2014 \text {. }\end{array}$ & $\begin{array}{l}\text { - Jugadores masculinos realizan entre } 14 \text { y } 24 \\
\text { cambios de posición ofensiva. } \\
\text { - Jugadores centrales se enfocan en la táctica } \\
\text { defensiva. }\end{array}$ \\
\hline $\begin{array}{l}\text { Monezi et al. } \\
(\underline{2018})\end{array}$ & $\begin{array}{lr}\mathrm{N}=2 \text { partidos de } \\
\text { Goalball (final, } & \text { 3er } \mathrm{y} \\
4^{\circ} & \text { lugar) } \\
\mathrm{N}= & 365 \\
\text { lanzamientos. } & \end{array}$ & $\begin{array}{l}\text { Analizar el tiempo- } \\
\text { movimiento de los } \\
\text { jugadores de Goalball. }\end{array}$ & $\begin{array}{l}\text { Análisis de vídeo } \\
\text { mediante } \\
\text { seguimiento manual } \\
\text { por un evaluador } \\
\text { experto. }\end{array}$ & $\begin{array}{l}\text { - Jugadas de banda duran más tiempo } \\
(2,54 \pm 2,72 \mathrm{~s}) \text {, cubren mayor distancia } \\
(1,65 \pm 2,88 \mathrm{~m}) \text { y tienen mayor velocidad, tanto } \\
\text { en la preparación de ataque }(0,59 \pm 0,77 \mathrm{~m} / \mathrm{s}) \\
\text { como en la fase de lanzamiento }(1,67 \pm 0,51 \\
\mathrm{m} / \mathrm{s}) \text {. } \\
\text { - Los jugadores centrales recorren distancias } \\
\text { cortas en la fase de lanzamiento }(1,89 \pm 2,22 \\
\mathrm{m}) \text { preocupándose más por la transición } \\
\text { defensiva. }\end{array}$ \\
\hline
\end{tabular}


- Lanzamiento entre piernas y giro, cubren mayores distancias en la preparación del ataque $(1,44 \pm 1,12$ y $2,27 \pm 2,99 \mathrm{~m}$ respectivamente).

Fuente: Elaboración propia. 
Tabla 4.

Artículos que presentaron Aspectos Psicológicos en Goalball

\begin{tabular}{|c|c|c|c|c|}
\hline Autor & Muestra & Objetivo & Metodología & Resultado \\
\hline Larsen (2014) & $\begin{array}{l}\mathrm{N}=\quad 6 \quad \text { atletas } \\
\text { Paralímpicas. }\end{array}$ & $\begin{array}{l}\text { Proporcionar } \\
\text { perspectivas aplicadas y } \\
\text { una descripción } \\
\text { detallada de un programa } \\
\text { de Entrenamiento de } \\
\text { Habilidades Mentales de } \\
\text { seis pasos. }\end{array}$ & $\begin{array}{l}\text { El programa tuvo una } \\
\text { duración de } 6 \text { meses. El } \\
\text { psicólogo deportivo se reunió } \\
\text { con jugadores dos veces al } \\
\text { día, para un total de } 60 \text { horas } \\
\text { de contacto. } \\
\text { Paso 1: reunión preliminar } \\
\text { con los entrenadores y la } \\
\text { asociación, Paso 2: inicio del } \\
\text { programa, Paso } 3 \text { : } \\
\text { mantenimiento del proceso y } \\
\text { motivación, Paso } 4: \\
\text { excitación y preparación para } \\
\text { la competencia, Paso } 5: \text { crear } \\
\text { una mentalidad ganadora y } \\
\text { un equipo fuerte, Paso } 6 \text { : } \\
\text { preparación para los } \\
\text { campeonatos. }\end{array}$ & $\begin{array}{l}\text { - Reflexiones de los participantes del } \\
\text { programa: en campeonato de } \\
\text { Europa, resultan ganadoras, y } \\
\text { expresan la importancia de haber } \\
\text { pertenecido al programa, mayor } \\
\text { conciencia de escucharse y mejor } \\
\text { comunicación verbal } \\
\text { proporcionando seguridad. Para los } \\
\text { entrenadores, las jugadoras fueron } \\
\text { más conscientes y concentradas } \\
\text { dentro de la cancha. } \\
\text { - Reflexiones de los psicólogos } \\
\text { deportivos: se logró el } \\
\text { funcionamiento del grupo, la } \\
\text { cohesión del equipo y la capacidad } \\
\text { para hacer frente a la adversidad. }\end{array}$ \\
\hline
\end{tabular}

Fuente: Elaboración propia. 


\section{DISCUSIÓN}

El objetivo de esta revisión fue describir el conocimiento científico producido en los últimos 10 años, respecto a la preparación deportiva, con énfasis en el sistema de entrenamiento en atletas de Goalball de diferentes niveles competitivos. En cuanto a los principales resultados, destacan investigaciones sobre los aspectos físicos que indican que pueden incidir positiva o negativamente en los atletas. En relación a los aspectos técnicos, se evidencia que existen diferencias en la técnica entre hombres y mujeres, el tipo de lanzamiento y la efectividad de este. Por otra parte, los aspectos tácticos estarán condicionados a la posición del atleta en el área de juego de Goalball. Finalmente, los aspectos psicológicos de los atletas, específicamente, el entrenamiento mental, se relaciona con un rendimiento eficiente. Las discusiones de cada uno de estos aspectos se presentan a continuación.

\section{Aspectos Físicos en Goalball}

Los estudios proporcionaron datos a través de metodologías como la filmación, análisis de vídeo, aplicación de pruebas físicas, test y antropometría (protocolo ISAK) para determinar, entre otros aspectos, la influencia de tiempo de reacción en la eficiencia de las defensas, aptitud cardiovascular, composición corporal, morfología, rendimiento de juego en relación a la discapacidad y niveles de equilibrio estático según experiencia, cargas de entrenamiento y excelencia deportiva. En el estudio de Valdés et al. (2014), se realizaron mediciones antropométricas, determinando que los atletas de Goalball de la región de la Araucanía (Chile) presentan un somatotipo mesoendomorfo con un porcentaje de grasa corporal (\%GC) de $28,78 \pm 8,21 \%$ y un $42,71 \%$ de masa muscular; sin embargo, aunque evaluaciones como la altura y el \%GC son importantes para determinar el somatotipo de los atletas de Goalball (Romanov et al., 2017), las variables antropométricas y de composición corporal no son determinantes en el lanzamiento del balón de Goalball (Campos et al., 2020), así como tampoco lo es la medición de masa corporal, altura del cuerpo, envergadura y longitud del cuerpo, en el rendimiento competitivo (Molik et al., 2015), algo que difiere con las investigaciones en deporte convencional (Quintero, Orsatto, Pulgarin y Follmer, 2019).

En este sentido, es importante mencionar que los métodos utilizados para estimación de composición corporal son distintos, siendo algunos de mayor precisión, por ejemplo, para determinación del \%GC (Kohrt, 1998; Genton, Hans, Kyle y Pichard, 2002; Nunes et al., 2020; López, Vélez, García, Arango, 2021), lo que puede influir en la diferencia de los 
resultados de los estudios, por lo que, las estimaciones de composición corporal en el marco de los aspectos físicos de la PD, requieren de una planificación y consideración de aspectos individuales de los atletas, pero entendiendo que es parte de un sistema (Zakharov, 2003). Por otra parte, aun cuando la repetición de movimientos y la disposición para la acción, disminuyen el tiempo de reacción, no se encontró diferencia de tiempo de reacción para acciones defensivas o en gol (Silva et al., 2010), existiendo factores individuales como, por ejemplo, fatiga cognitiva, edad, experiencia, posición del jugador, expectativa, incertidumbre y capacidad visual, que si afectan la reacción de los jugadores (Reid et al., $\underline{2020})$.

En relación a la aptitud cardiovascular, Gulick y Malone (2011) evidenciaron fuerte correlación $(r=0,77)$ entre la prueba en ergómetro y la prueba de sonido modificada, demostrando que esta última, es válida para determinar aptitud cardiovascular.

En esta línea, la aplicación de pruebas físicas de campo en atletas de Goalball, como el salto de contramovimiento (CMJ), arroja correlaciones inversamente proporcionales y significativas con \%GC ( $r=-0,79)$ y también con Yoyo Test $(r=-0,90)$, asimismo, el test CMJ demostró una alta correlación con la velocidad de lanzamiento $\left(R^{2}=0,57\right)$, que refleja una alta correlación frente a la prensión manual $(r=0,66)$ (Goulart-Siqueira et al., 2018). Cabe destacar lo referido por Campos et al. (2020), quienes determinaron la asociación y relación entre fuerza máxima y potencia muscular, identificando correlaciones inversamente proporcionales y significativas entre Test de Lanzamiento y $1 R M e(r=-0,80)$ y una correlación alta y significativa entre lanzamiento con balón medicinal (AMB) y $1 \mathrm{RMe}(\mathrm{r}=$ 0,89 ) pudiendo ser utilizada la prueba AMB para estimar la fuerza máxima en los atletas. Si realizamos una analogía con el deporte convencional, existen diferencias en la evidencia encontrada, como, por ejemplo, los hallazgos de Franceschi, Conte, Airale y Sampaio (2020) quienes destacan probables puntuaciones más altas en el rendimiento en salto durante la fase competitiva junto a una fatiga perceptiva más baja; lo anterior, relacionado a un sistema de monitoreo de los atletas.

Otro aspecto interesante en esta investigación, es que se identifica el uso de la tecnología, lo que permitió a Bednarczuk et al. (2017), a través de la utilización de una plataforma estabilográfica, determinar que existe un mayor equilibrio estático en atletas con experiencia de hasta 10 años de entrenamiento, no así en aquellos que llevan más de 10 años entrenando; identificando, además, que los jugadores que participaron de la fase final de los Juegos Paralímpicos 2012, poseen mayor estabilidad postural. 
Entre las investigaciones referidas al componente de rendimiento físico, se evidencian relaciones entre parámetros fisiológicos obtenidos en laboratorio y juegos simulados o el rendimiento técnico del juego (Alves et al., 2018), existiendo correlaciones muy probables entre el VO2máx en condición de laboratorio con la frecuencia de lanzamiento $(r=0,87)$ y la recuperación en los juegos $(r=0,9)$; destacando la importancia del metabolismo aláctico, al determinar una correlación muy probable entre la contribución aláctica y la densidad de acción $(r=0,95)$.

\section{Aspectos Técnicos en Goalball}

Los estudios proporcionaron datos a través de filmaciones y análisis de videos de campeonatos de nivel mundial y paralímpico, por género (hombres y mujeres) identificando que, en el caso de los hombres, los tiros de rotación de $360^{\circ}$ con una mano y, en las mujeres, lanzamientos lisos con una mano y en carrera, fueron los más ejecutados, debido a que la carrera y el giro implementa velocidad al balón (Almeida et al., 2008 citado en Amorim, Botelho, Sampaio, Molina, y Corredeira, 2010). No obstante, la técnica es un factor determinante en la efectividad del lanzamiento, es por eso que la trayectoria escogida en la técnica utilizada, influye grandemente en el éxito del lanzamiento (Morato et al., 2018; Amorim et al., 2010)

En ese sentido, en los atletas masculinos se evidencia mayor porcentaje de lanzamientos hacia el sector lateral en paralelo y en las mujeres, los lanzamientos entre las posiciones central y lateral con trayectoria en paralelo (Link y Weber, 2018), aunque, tanto hombres como mujeres fueron más efectivos en lanzamientos curvos, siendo importante la reducción del tiempo de lanzamiento para determinar la efectividad de un gol (Morato et al., 2018).

La técnica ofensiva y defensiva, así como sus fases (posición de expectativa, acciones de desplazamiento corporal o la posición final e impacto) deben ser practicados y entrenados en detalle, como lo confirman Nascimento y Camargo (2012), así como también, las características propias de hombres y mujeres en relación a capacidades físicas las que van a incidir en los aspectos de técnica.

\section{Aspectos Tácticos en Goalball}

El ciclo de defensa se equilibra mediante retroalimentación negativa o positiva, la que va a generar un nuevo estándar organizativo (Morato et al., 2012). En ese sentido, Morato et al. (2012) declara importante considerar que la estructura y organización deben ir 
correlacionadas (terreno de juego, equipos, jugadores, balón, porterías) ya que, si se modifica el patrón organizativo y patrón organizacional, (invasión territorial, mayor número de jugadores dimensiones de cancha, entre otros) tendríamos un deporte distinto a lo que hoy conocemos como Goalball.

El ciclo del juego presenta la defensa del balón como resultado estándar, que tiende a equilibrarse mediante mecanismos de retroalimentación negativa, no obstante, cuando el resultado de la defensa se ve modificada producto de un gol, penalti o infracción, puede haber una bifurcación que provoca un nuevo estándar organizativo (Morato et al., 2012). En esa línea, se reflejan las investigaciones de Kornev y Pavdrov (2017) y Monezi et al. (2018), en donde los atletas presentan una táctica organizativa que permite a los jugadores centrales realizar un mayor número de acciones defensivas y, en la táctica ofensiva, los jugadores de banda son los encargados de liderar el ataque, generando patrones organizacionales restrictos que pueden ser practicados en los entrenamientos utilizando estructuras que simulen ese patrón (Morato et al., 2012).

Monezi et al. (2018) encontraron que los jugadores laterales, en la fase ofensiva, son quienes cubren mayor distancia $(1,65 \pm 2,88 \mathrm{~m})$ y a mayor velocidad $(0,59 \pm 0,77 \mathrm{~m} / \mathrm{s})$, tanto en la preparación de lanzamiento, como en el ataque. Los cambios de dirección realizados por los jugadores, son un aspecto táctico clave que implica al equipo rival generar confusiones en el ciclo auto-organizacional, siendo utilizados en los equipos ganadores de la final masculina del Mundial de Goalball 2014, realizando, entre 14 y 24 cambios de posición ofensiva (Kornev y Pavdrov, 2017).

Es necesario considerar la táctica a nivel competitivo, y, en ese sentido, Amorim et al. (2010) analizaron las acciones ofensivas de equipos que competían a nivel nacional y equipos que competían a nivel europeo, evidenciando diferencias. Sin embargo, en ambos niveles competitivos optan por realizar acciones ofensivas desde las zonas laterales con trayectorias cortas (lanzamientos paralelos o diagonales cortas), siendo un aspecto táctico empleado por gran cantidad de atletas en los diversos estudios (Monezi et al., 2018; Kornev y Padrov, 2017; Morato et al., 2012; Morato, 2012), debido a que, según Morato et al. (2018) las trayectorias paralelas y diagonales cortas recorren menores trayectos, viéndose implementada la velocidad del balón, aumentando la probabilidad de éxito.

Estos resultados reflejan que, como deporte colectivo sin invasión, presenta ciertas acciones tácticas apropiadas a las características del juego. Tal como se manifiesta en Sitting Volleyball (voleibol sentado), el cual presenta un patrón organizacional similar al Goalball al ser un deporte colectivo sin invasión territorial, en donde los jugadores con 
discapacidad mínima ejecutan mayores cantidades de acciones de ataque, en cambio los atletas que presentaban amputaciones transfemorales, son atletas destinados a realizar acciones mayoritariamente defensivas manifestadas en el bloqueo del ataque contrario (Souza, Maraes, Gomes y Lamas, 2020). Por tanto, las características propias del atleta van a determinar la táctica empleada según los requerimientos tácticos que la modalidad deportiva demande.

Finalmente, en relación a aspectos tácticos en el Goalball, encontramos un estudio que no se incluye en nuestra revisión sistemática, pero que es fundamental conocer dentro de la modalidad, en donde los autores desarrollaron un sistema de observación para el análisis de partidos de Goalball mediante una planilla en base a seis orígenes de las áreas de juego, siendo un instrumento de gran importancia para los entrenadores y/o profesores enfocados en la evaluación de los aspectos técnicos, rendimiento físico, psicológico y, por sobre todo, para analizar los aspectos tácticos de la modalidad (Morato, da Cunha Furtado, Gamero, Magalhães, y de Almeida Gavião, 2016).

\section{Aspectos Psicológicos en Goalball}

Pocos autores han discutido cómo un psicólogo deportivo puede trabajar con atletas con discapacidad (Hanrahan, 1998), y la mayor parte de la literatura se centra en el desarrollo de habilidades mentales específicas (Martin, 1999, citado en Larsen, 2014). En esta investigación se encontró un artículo en el área de psicología, referido a la aplicación de un programa de entrenamiento de habilidades mentales en alto rendimiento, obteniendo reflexiones por parte del entrenador y jugadoras destacando la importancia de haber sido parte de este programa, favoreciendo su consciencia, comunicación verbal y, a decir de los entrenadores, mayor conciencia y concentración durante la competencia. Por otra parte, los psicólogos deportivos del equipo hacen referencia al funcionamiento, cohesión y capacidad de hacer frente a la adversidad, lo que finalmente les permitió, ser Campeonas de Europa y clasificar a los Juegos Paralímpicos de Londres 2012.

Existe una carencia de estudios enfocados en este ámbito, más aún en las modalidades paralímpicas. Se ha demostrado que la variable psicológica influye positiva o negativamente en el funcionamiento físico, técnico y táctico y, por tanto, en el rendimiento deportivo de un atleta (Buceta, 1998). Así, un entrenamiento mental le permite al atleta adquirir una serie de estrategias para desarrollar habilidades psicológicas que les ayuden a enfrentar los entrenamientos y competencias de la mejor forma posible, como los atletas 
de élite que suelen utilizar habilidades mentales para lograr un rendimiento eficaz (Dosil, 2008).

Estos datos son corroborados por De la Vega, Galán, Ruiz y Tejero (2013) quienes evidencian en atletas de Boccia que las percepciones que ellos tienen sobre sus estados anímicos inciden en su rendimiento, siendo importante diferenciar la competencia individual de la colectiva, ya que se detecta un patrón estable de alteración no emocional, aunque con un grado de depresión estadísticamente mayor en la competencia colectiva en comparación con la competencia individual.

En esta dirección, es importante mencionar que existe un cuestionario de Características Psicológicas relacionadas al Rendimiento Deportivo (CPRD), el cual contempla 5 escalas (Gimeno, Buceta y Pérez-Llanta, 2001), siendo utilizado en diversos estudios con atletas convencionales y también con atletas Paralímpicos. En este último caso, se aplicó el cuestionario CPRD previo a un campeonato nacional (Chile) a atletas de Boccia, evidenciando que la clasificación BC4 obtuvo mejores resultados en todas sus variables en comparación a las demás clases deportivas (Correa, Castelli, CamposCampos y Luarte, 2021).

Finalmente, se observa escasez de estudios en el área psicológica, siendo, por una parte, una limitación para establecer un perfil de los atletas y comprender sus percepciones frente a las competencias y por otra, un desafío y un vasto campo de oportunidades para estudios futuros.

\section{CONCLUSIÓN}

Los estudios seleccionados en esta revisión sistemática en relación a la PD en atletas de goalbal de diferentes niveles competitivos, más específicamente respecto al sistema de entrenamiento, indican que existen factores individuales que incidirán positiva o negativamente en el desempeño de los atletas y específicamente en el tiempo de reacción de los jugadores. Por otra parte, se deben plantear ejercicios enfocados al metabolismo aeróbico y anaeróbico, logrando un entrenamiento eficiente en la fuerza/potencia de extremidades superiores e inferiores. Por otro lado, resulta interesante las diferencias existentes entre la técnica de lanzamiento en la rama femenina y masculina, predominando la tendencia de un lanzamiento en giro $360^{\circ}$ en hombres y lanzamientos lisos con una mano y en carrera en mujeres, siendo la velocidad, un factor determinante en la probabilidad de marcar un gol. 
En este sentido, parece necesario tener en cuenta las conductas propias de la disciplina en función de la posición de los jugadores dentro de la cancha, debido a que los atletas centrales, son los que realizan mayor cantidad de acciones defensivas, en cambio, los atletas laterales son los encargados de liderar las acciones de ataque en un juego de Goalball. Para esto, es fundamental la comunicación verbal, que proporcionará seguridad, concentración y consciencia en el performance deportivo en los(as) atletas, aumentando la cohesión de equipo y la capacidad para hacer frente a la adversidad, logrando un rendimiento eficiente, a través de un entrenamiento mental planificado en la PD de los atletas de Goalball.

A modo de conclusión, este estudio buscó describir las evidencias científicas desarrolladas en la última década (2010-2020) respecto al sistema de entrenamiento (aspectos físicos, técnicos, tácticos y psicológicos) en atletas de Goalball de diferentes niveles competitivos, con el objetivo de generar información relevante para los profesionales que participan directamente con la modalidad, tanto en la preparación de nuevos atletas como en el desarrollo de jugadores ya experimentados. Se observa la necesidad de mayores evidencias y conocimientos acerca de la PD, principalmente de los aspectos relacionados al sistema de entrenamiento en Goalball ya que constituyen factores imprescindibles que deben ser contemplados por los entrenadores, en función de optimizar el rendimiento competitivo de sus atletas.

\section{REFERENCIAS}

Alves, I., Kalva-Filho, C., Aquino, R., Travitzki, L., Tosim, A., Papoti, M. y Morato, M. (2018). Relationships Between Aerobic and Anaerobic Parameters with Game Technical Performance in Elite Goalball Athletes. Frontiers in Physiology, 9(1636), 1-10. doi: https://doi.org/10.3389/fphys.2018.01636

Amorim, M., Botelho, M., Sampaio, E., Molina, J. y Corredeira, R. (2010). Caracterización de los patrones comportamentales de los atletas con discapacidad visual practicantes de Goalball. Revista Electrónica Interuniversitaria de Formación del Profesorado, 13(3), 47-57. Recuperado de https://www.redalyc.org/articulo.oa?id=217015214005

Bednarczuk, G., Molik, B., Morgulec-Adamowicz, N., Kosmol, A., Wiszomirska, I., Rutkowska, I. y Perkowski, K. (2017). Static balance of visually impaired Paralympic Goalball players. International Journal of Sports Science \& Coaching, 12(5), 611617. doi: https://doi.org/10.1177/17479554117727791 
Buceta, J. (1998). Psicología del entrenamiento deportivo. Madrid, España: Dykinson.

Campos, L. F., Campos-Campos, K., Gajardo-Oñate, J., Rocha, C., dos Santos, L. G., Plaza, R. y Lara, J. (2020). Caracterización de los deportistas Paralímpicos de Goalball chilenos y asociación entre variables Antropométricas, de Composición Corporal y Fuerza en el Lanzamiento del Balón. Retos, 38(38), 41-44. doi: https://doi.org/10.47197/retos.v38i38.73067

Campos, L.F., da Luz, L., Rocha, C., Nogueira, C., Roca, V. y Gorla, J. (2019). Validación de estudios de prueba para el análisis de la potencia aeróbica en atletas tetrapléjicos. Apunts: Educación Física y Deportes, (135), 68-81. doi: http://dx.doi.org/10.5672/apunts.2014-0983.es.(2019/1).135.05

Correa, M., Castelli, L.F., Campos-Campos, K. y Luarte, C. (2021). Características Psicológicas del Rendimiento Deportivo en atletas de Boccia pertenecientes al Comité Paralímpico de Chile. Revista Peruana de Ciencias de la Actividad Física y el Deporte, 8(2), 1139-1146. doi: https://rpcafd.com/index.php/rpcafd/article/view/138/186

Da Costa Santos, C., de Mattos Pimenta, C. y Nobre, M. (2007). A estratégia PICO para a construção da pergunta de pesquisa e busca de evidências. Revista LatinoAmericana de Enfermagem, 15(3). doi: https://doi.org/10.1590/S010411692007000300023

Dosil, J. (2008). Psicología de la actividad física y del deporte. España: McGraw-Hill.

Franceschi, A., Conte, D., Airale, M. y Sampaio, J. (2020). Training Load, neuromuscular readiness and perceptual fatigue profile in youth elite long- jump athletes. International Journal of Sports Physiology and Performance, 15(7), 1034-1038. doi: https://doi.org/10.1123/ijspp.2019-0596

Genton, L., Hans, D., Kyle, U. G. y Pichard, C. (2002). Dual-Energy X-ray absorptiometry and body composition: differences between devices and comparison with reference methods. Nutrition, 18(1), 66-70. doi: https://doi.org/10.1016/s08999007(01)00700-6

Gimeno, F., Buceta, J. M. y Pérez-Llanta, M. (2001). El Cuestionario "Características psicológicas relacionadas con el rendimiento deportivo" (CPRD): Características Psicométricas. $\quad$ Análise $\quad$ Psicológica, 119), http://www.scielo.mec.pt/pdf/aps/v19n1/v19n1a09.pdf

Gomes, A. (2009). Treinamento desportivo: Estruturação e periodização (2a ed.). Brasil: Artmed. 
Goulart-Siqueira, G., Benítez-Flores, S., Ferreira, A., Zagatto, A., Foster, C. y Boullosa, D. (2018). Relationships between Different Field Test Performance Measures in Elite Goalball Players. Sports, 7(1), 1-7. doi: https://doi.org/10.3390/sports7010006

Gulick, D. y Malone, L. (2011). Field Test for Measuring Aerobic Capacity in Paralympic Goalball Athletes. International Journal of Athletic Therapy \& Training, 16(5), 22-25. doi: https://doi.org/10.1123/ijatt.16.5.22

Hanrahan, S. J. (1998). Practical considerations for working with athletes with disabilities. The Sport Psychologist, 12(3), 346-357. doi: https://doi.org/10.1123/tsp.12.3.346

International Blind Sports Federation [IBSA]. (2020). Goalball - General information. Recuperado de https://www.ibsasport.org/sports/goalball/

Kohrt, W. (1998). Preliminary evidence that DEXA provides an accurate assessment of body composition. Journal of Applied Physiology, 84(1), 372-377. doi: https://doi.org/10.1152/jappl.1998.84.1.372

Kornev, A. y Pravdov, M. (2017). Goalball Game Techniques and Tactics. Teoriya I Praktika Fizicheskoy Kultury, (4), 20-22. Recuperado de https://www.researchgate.net/publication/317026656 Goalball game techniques and tactics

Larsen, C. (2014). Preparing for the European Championships: A Six-step Mental Skills Training Program in Disability Sports. Journal of Sport Psychology in Action, 5(3), 186-197. doi: https://doi.org/10.1080/21520704.2014.971989

Link, D. y Weber, C. (2018) Finding the gap: An empirical study of the most effective shots in elite goalball. PLoS ONE, 13(4), e0196679. doi: https://doi.org/10.1371/journal.pone.0196679

López, A., Vélez, J., García, A., Arango, E. (2021). Concurrent validity of five prediction equations to evaluate fat percentage in a sports group expected to yield high performace from the city of Medellin - Colombia. Biomédica, 41(1). doi: https://doi.org/10.7705/biomedica.5333

Matveev, L. (1996). Preparação Esportiva. Brasil: Livraria Aratebi Ltda.

Moher, D., Liberati, A., Tetzlaff, J., Altman, D., Altman, D., The PRISMA Group. (2009). Preferred reporting items for systematic reviews and meta-analyses: The PRISMA statement. PLoS Medicine, 6(7), e1000097. doi: https://doi.org/10.1371/journal.pmed.1000097

Molik, B., Morgulec-Adamowicz, N., Kosmol, A., Perkowski, K., Bednarczuk, G., Skowronski, W., Gomez, M., Koc, K., Rutkowska, I. y Szyman, R. (2015). Game 
Performance Evaluation in Male Goalball Players. Journal of Human Kinetics, 48(1), 43-51. doi: https://doi.org/10.1515/hukin-2015-0090

Monezi, L., Magalhães, T., Morato, M., Mercadante, L., da Cunha Furtado, O. y Misuta, M. (2018). Time-motion analysis of Goalball players in attacks: differences of the player positions and the throwing techniques. Sports Biomechanics, 18(5), 470-481. doi: https://doi.org/10.1080/14763141.2018.1433871

Morato, M. (2012). Análise do jogo de Goalball: Modelação e interpretação dos padrões de jogo nas Paralimpiadas de Pequim 2008 (Tese de Doutorado). Universidade Estadual de Campinas, Brasil. Recuperado de http://repositorio.unicamp.br/jspui/handle/REPOSIP/275036

Morato, M. y Gavião, J. (2012). Goalball Esporte Paralímpico. Brasil: Atheneu.

Morato, M., da Cunha Furtado, O., Gamero, D., Magalhães, T. y de Almeida Gavião, J. (2016). Development and evaluation of an observational system for Goalball match analysis. Revista Brasileira de Ciências do Esporte, 39(4), 398-407. doi: https://doi.org/10.1016/j.rbce.2016.08.002

Morato, M., Menezes, R., Fonseca, S. y da Cunha Furtado, O. (2018). Faster balls increase the probability of scoring a goal in female and male elite Goalball. Revista Brasileira de Ciências do Esporte, 40(4), 427-434. doi: https://doi.org/10.1016/j.rbce.2018.03.027

Morato, M., Simöes, M., y Gavião, J. (2012). Os processos auto-organizacionais do goalball. Revista Brasileira de Ciências do Esporte, 34(3), 741-760. doi: https://doi.org/10.1590/S0101-32892012000300015

Moreira, A. (2010). La periodización del entrenamiento y las cuestiones emergentes: el caso de los deportes de equipo. Revista Andaluza de Medicina del Deporte, 3(4). Recuperado

de https://ws072.juntadeandalucia.es/ojs/index.php/ramd/article/view/363

Nascimento, D. y Camargo, W. (2012). Decodificando o Goalball para Professores-Técnicos de Educação Física Adaptada: fundamentos técnicos. Cadernos de Formação RBCE, 3(1), 57-74. Recuperado de http://revista.cbce.org.br/index.php/cadernos/article/view/1285/763

Nunes, R., Bezerra, E., Orssatto, L., Moreno, Y., Loturco, I., Duffield, R., Silva, D. y Guglielmo, L. (2020). Assessing body composition in rugby players: agreement between different methods and association with physical performance. Journal of 
Sports Medicine and Physical Fitness, 60(5), 733-742. doi: https://doi.org/10.23736/S0022-4707.20.10487-0

Parsons, A. y Winckler, C. (2012). Esporte e a pessoa com deficiência: contexto histórico. En T. Mello y C. Winckler (Eds.), Esporte Paralímpico (pp. 3-14). Brasil: Atheneu.

Petrigna, L., Giustino, V., Zangla, D., Aurea, S., Palma, R., Palma, A., Battaglia, G. (2020). Physical fitness assessment in goalball: A scoping Review of the Literature. Heliyon, 6(7), e04407. doi: https://doi.org/10.1016/j.heliyon.2020.e04407

Platonov, V. (2001). Teoría general del entrenamiento Deportivo Olímpico. Badalona, España: Paidotribo.

Platonov, V. (2008). Tratado Geral de Treinamento Desportivo. Brasil: Phorte.

Quintero, A., Orsatto, L., Pulgarin, R. y Follmer, B. (2019). Physical Performance, Body Composition and Somatotype in Colombian Judo Athletes. Ido Movement for Culture. Journal of Matrial Arts Anthropology, 19(2), 56-63. doi: https://doi.org/10.14589/ido.19.2.8

Reid, B., Schreiber, K., Shawhan, J., Stewart, E., Burch, R. y Reimann, W. (2020). Reaction time assessment for coaching defensive players in NCAA division 1 American football: A comprehensive literature review. International Journal of Industrial Ergonomics, 77, 102942. doi: https://doi.org/10.1016/j.ergon.2020.102942

Da Rocha, M. (2007). Treinamento de força aplicado ao Goalball (Trabalho de Conclusão de Curso Especialização. Universidade Estadual de Campinas, Brasil. Recuperado de http://www.bibliotecadigital.unicamp.br/document/?code=000437296\&opt=1

Roever, L. (2017). Understanding Sistematic Review Study. Revista da Sociedade Brasileira de Clínica Médica, 15(2), 127-130. Recuperado de https://docs.bvsalud.org/biblioref/2017/11/875614/152 127-130.pdf

Romanov, R., Medovic, B. Stupar, D., Jezdimirovic, T. y Garunovic, B. (2017). The Connection Between Certain Morphological Parameters and Results in Goalball Players. International Journal of Morphology, 35(4), 1396-1402. doi: http://doi.org/10.4067/S0717-95022017000401396

Santos, L.G. (2019). Desempenho dos finalistas do campeonato mundial masculino de paracanoagem e proposta de indicadores para o controle do treinamento (Tese de Doutorado). Universidade Estadual de Campinas, Brasil.

Silva, G., Pereira, V., Deprá, P. y Gorla, J. (2010). Tempo de reação e a eficiência do jogador de Goalball na interceptação/defesa do lançamento/ataque. Motricidade, 6(4), 1322. Recuperado de http://www.scielo.mec.pt/pdf/mot/v6n4/v6n4a03.pdf 
Souza, B., Maraes, V., Gomes, M. y Lamas, L. (2020). Seated Volleyball: The Influence of Motor Deficiency Type on Players' Tactical Roles. Revista Brasileira de Medicina do Esporte, 26(4), 342-346. doi: https://doi.org/10.1590/1517-869220202604218192

Souza, L.G., Barra, C., Fernández, M., Santos, L.G., Casteleti, J.P., Luarte, C. y Campos, L.F. (2020). Limitaciones y posibilidades en el entrenamiento de baloncesto en silla de ruedas. Revista Peruana de Ciencias de la Actividad Física y el Deporte, 7(4), 1036-1044.

Recuperado

de https://www.rpcafd.com/index.php/rpcafd/article/view/117

Valdés, P., Godoy, A. y Herrera, T. (2014). Somatotipo, Composición Corporal, Estado Nutricional y Condición Física en Personas con Discapacidad Visual que Practican Goalball. International Journal of Morphology, 32(1), 183-189. Recuperado de https://scielo.conicyt.cl/pdf/ijmorphol/v32n1/art31.pdf

de la Vega, R., Galán, A., Ruiz, R. y Tejero, C.M. (2013). Estado de ánimo precompetitivo y rendimiento percibido en Boccia Paralímpica. Revista de Psicología del Deporte, 22(1), 39-45. Recuperado de https://www.redalyc.org/pdf/2351/235127552006.pdf

Zakharov, A. (2003). Ciência do Treinamento Desportivo ( $2^{a}$ ed.). Brasil: Grupo Palestra esporte. 